\title{
Personalized Hand Pose and Gesture Recognition System for the Elderly
}

\author{
Mahsa Teimourikia, Hassan Saidinejad, Sara Comai, and Fabio Salice \\ Department of Electronics, Information and Bioengineering \\ Politecnico di Milano \\ via Ponzio 34/5, 22100, Milan, Italy
}

\begin{abstract}
Elderly population is growing all over the globe. Novel human-computer interaction systems and techniques are required to fill the gap between elderly reduced physical and cognitive capabilities and the smooth usage of technological artefacts densely populating our environments. Gesture-based interfaces are potentially more natural, intuitive, and direct. In this paper, we propose a personalized hand pose and gesture recognition system (called HANDY) supporting personalized gestures and we report the results of two experiments with both younger and older participants. Our results show that by sufficiently training our system we can get similar accuracies for both younger and older users. This means that our gesture recognition system can accommodate the limitations of an ageing-hand even in presence of hand issues like arthritis or hand tremor.
\end{abstract}

Keywords: gestural interaction, gesture recognition system, elderly.

\section{Introduction}

According to demographic studies, elderly population is growing all over the globe. This demographic shift is a by-product of lower fertility and better health conditions leading to lower mortality among older persons. The number of older persons aged 60 or over is projected to be 2 billion in 2050, three times the number in 2000, comprising 22 percent of the world population [1. It is believed that population ageing will have significant socio-economic consequences for which a preparation is needed [2]. The WHO Active Ageing framework [3] considers health, safety, independence, mobility, and participation as the five higher level needs of the older persons for a higher quality of life. The important role ICT can play in this context is widely recognized [4. For instance, AAL (Ambient Assisted Living) is a joint European project focused on the usage of ICT to help older persons in ageing well and is supported by the biggest EU Research and Innovation program, Horizon 2020.

For the realization of the inclusive society of future in which age and capability related discriminations are lifted and the older persons have the same opportunities as others, "Design for All" plays a central role. It concerns the design of products, services, and applications with accessible and adaptable interfaces for

C. Stephanidis and M. Antona (Eds.): UAHCI/HCII 2014, Part III, LNCS 8515, pp. 191-202, 2014.

(C) Springer International Publishing Switzerland 2014 
special users like the elderly 4. As far as the interfaces are concerned, novel human-computer interaction systems and techniques are required to fill the gap between elderly reduced physical and cognitive capabilities and the smooth usage of technological artefacts increasingly populating their environment.

Gesture-based interfaces have been around for several years and are considered to be potentially more natural, creative, and intuitive [5]. Gesticulations could be done with different body parts. [6] states that the hand is the most effective part of the body for communication through gestures. [7] supports this idea by analysing the gesturing literature and finding that hand-based gestures are at the top. [8] lists a set of requirements for effective hand-gesture interfaces which indicate two major research challenges: one concerns technical issues on the machine side to guarantee responsiveness, recognition accuracy, gesture spotting, etc; the other concerns interaction design issues on the human side like the gesture vocabulary size, learnability, comfort, etc.

Gesture-based interaction design is even more challenging for elderly people mainly due to their reduced physical and cognitive capabilities. Effects of ageing on sensory modalities, perception, cognition, and movement control are described in [9]. One of the challenges of hand gesture-based interfaces for the elderly lies in confronting the impacts of ageing on the hand. Research and studies in geriatrics suggest that for elderly people, both men and women, degenerative changes in musculoskeletal, vascular, and nervous systems lead to hand function degradation in terms of handgrip and finger-pinch strength, maintaining pinch force and posture, and dexterity of manual movements 10. Other challenges include hand tremor which concerns involuntary shaking of the hand, and joint pains due to arthritis. These restrictions in hand functionality could impede an effective interaction with the interface. However, despite these age-related issues, studies like 11, comparing younger and older participants, suggest that age is not an exclusion factor for gestural interaction. We think that personalization could make gestural interaction more accessible and adaptable to some agerelated issues, especially for an ageing hand.

In this work, we propose a vision-based hand gesture recognition system leveraging intuitive and natural poses and gestures of the hand that can be personalized. This system (called HANDY) is flexible enough to be trained (with a small number of trainings) for a variety of hand poses and gestures that meet the user's specific needs. In order to evaluate the system, we conducted two experiments: the first one on younger adults and the second one on older adults. We measured recognition accuracy of the system in both cases and qualitatively evaluated the difficulty of hand pose creation and gesture performance. Our results show that by sufficiently training our system we can get similar accuracies for both younger and older users. This means that our gesture recognition system can accommodate the limitations of an ageing-hand even in presence of hand issues like arthritis or hand tremor.

The following parts of the paper are structured as follows. In Sect. 2 we briefly review the work which has been done in the areas of gesture recognition systems, gesture interaction design, and gesture interaction and its applications for the 
elderly. Section 3 introduces the different parts of our gesture recognition system (HANDY). Section 4 is devoted to the description of our two experiments on younger and older adults. The results of the evaluation are discussed in Sect. 5 . Finally, in Sect. 6. we present our conclusions and our future research path.

\section{Background and Related Work}

\subsection{Hand Gesture Recognition Systems}

Gesture recognition methods fall into two broad categories: wearable-based and vision-based [12. Data glove is an example of a wearable sensor (e.g., [13]) which can provide accurate measurements of hand pose and movements. However, wearable sensors are commonly costly and intrusive. Vision-based techniques can be divided into two broad approaches [14]: model-based approaches that take advantage of a 3D or $2 \mathrm{D}$ model of the hand and appearance-based approaches which are used to extract the features of the visual data for gesture recognition. Model-based approaches usually suffer from high complexity in implementation and cannot be used in live applications. Appearance-based approaches use RGB or depth data or both as input. Our framework falls into this category. In our system, we will take advantage of the body tracking information extractable from Kinect SDK, so that depth thresholding (to locate and segment the hand) can be done regardless of the position of the hand.

Zhu and Pun [15] use Kinect depth data for extracting the trajectory data sequence of the hand movements, but, do not consider hand postures. In a similar way, [16] and [17] introduce a gesture recognition approach that considers the motion and shape information of the hand using depth data. However, the hand shape is considered to remain the same during the gesturing and pose estimation is done once at the beginning of the gesture. In another study by Chen et al. 18. HMM continuous gesture recognition is proposed considering the spatial and temporal features of the gestures. Yet, a small number of poses is recognized in this approach and the posture of the hand does not change while performing the gesture. HMM has been adopted also in the work by Starner et al. [19] for American Sign Language (ASL) recognition. To recognize the sign language they have ignored the detailed shape and pose of the hand and have only considered, coarse hand pose, orientation, and the trajectory of the gesture through time, and used such information as input for the recognition system. In a more recent work, Molina et al. 20. proposed an approach for static pose estimation and dynamic gesture recognition. They successfully recognized the gestures that include the change in the hand postures. They obtained an accuracy of $90 \%$ for recognition of combination of static hand postures and dynamic gestures.

Differently from these approaches, we consider hand poses and gestures composed of their combinations: hand poses are modeled by their skeleton, and time series analysis algorithms and HMM techniques are used to recognize sequences of hand poses. Using these techniques also personalized gestures can be defined and more flexibility can be added in case of gesture evolution. 


\subsection{Gestural Interaction for the Elderly}

In general, gestural interaction was recognized as an important form of communication already in early studies (e.g., [21]). There has been a lot of research studying 3D spatial hand gestures. For instance, 22 studies freehand pose-based gestural interaction for novice users. 23] conducts a user study to understand users' preferences to manipulate digital content on a distant screen. Gestures represent an important aspect towards Natural User Interfaces. However, according to 24] even if gestures represent a useful addition in the interaction, many gestures are neither natural nor easy to learn or remember. Also 25] believes that the currently available systems are not natural, since they require to learn predefined artificial gestures, typically depending on the device or the application. This makes specific users like elderly reluctant to approach such applications.

As for the elderly, the very first question to face with is whether gestural interaction is even suitable for them taking into account their age-related sensory, motor, and cognitive impairments. In [11, authors describe the results of their devised experiment in which they compare the performance of younger and older participants in terms of accuracy and speed for a set of 42 one finger touch gestures. Their findings show a significant impact of the age only on the speed and not on the accuracy of the performance. And thus, they conclude that there is not anything intrinsic to gestural interaction which prevents the elderly from using it. They mention some motor problems from the literature which could be potential barriers for gestural interaction for older users: reduced wrist flextion and extension, less efficient perceptual feedback system, not having enough force for quick movements, having more submovements, and problems in performing continuous movements and movement coordination.

There are different application domains that could benefit from gestural interfaces. Gesture-based interfaces in smart homes for elderly with mobility impairment is one of the applications. Authors of [26] present an implemented gesture-based interface for elderly people for communication with an assistive robot in the context of a smart house. Defining six word signs (defined based on baby signs), using a monitor-mounted webcam, colored gloves, and Hidden Markov Models for gesture recognition, they claim to reach a recognition accuracy of $94.33 \%$. Exergame is another potential application of gestural interaction for elderly that mainly focuses on physical well-being and rehabilitation. In [27, authors have developed two mobile games for the elderly using 3D gestural interaction for rehabiliation purposes. They concluded that gesture recognizer performance in terms of spotting quick gestures and ignoring unintended ones plays an important role in usability measures. Moreover, they consider familiarity and naturalness as two factors important in engaging older users in mobile gestural games. In another study [28, authors present the results and findings of two studies concerning gesture set design for a full-body motion-based game for institutionalized elderly people. They argue that user specific abilities are usually ignored by game designers. So, considering physical limitations, intuitiveness of the gestures, and learning easiness, they suggest a gesture set comprised of 


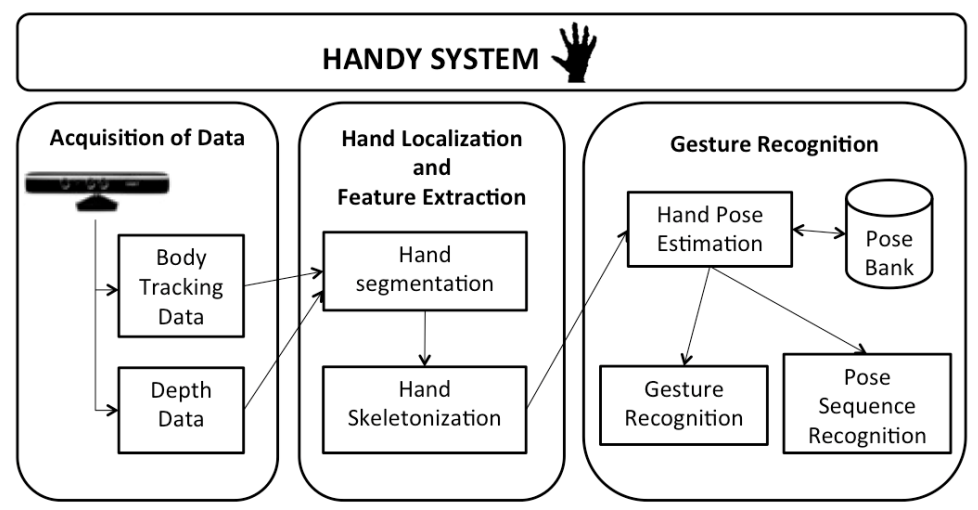

Fig. 1. The HANDY Gesture Recognition System

four static and four dynamic gestures. Discussing the results, they conclude that adaptability of the interaction is essential because of the heterogeneous participant abilities, either physical or cognitive. Finally, gestural interaction could be used in multimodal interfaces, especially those involving speech and gesture. In [29], authors describe a user study concerning speech and gesture interaction. The study takes place in an ambient assisted living lab with an intelligent wheelchair for assisted mobility and smart furniture for device control.

Our work is independent from the applications: it focusses on the possibility of personalizing the gestures taking into account also the characteristics of the hands of the elderly.

\section{The Handy System}

Figure 1 depicts the architecture of the HANDY system for hand gesture recognition. The description of the different parts of the architecture follows. A more detailed explanation of our gesture recognition system is presented in [30].

\subsection{Hand Localization and Segmentation}

This part of the system is responsible for locating the position of the hand and tracking it through time. RGB and depth information are extracted from Kinect sensors to locate and track the hand. NiTE skeletal tracking is used which gives the position of the wrist and the center of the hand. This information is used to perform hand segmentation with depth thresholding. Depth thresholding is an easy and quick way for real-time hand segmentation and it can be greatly beneficial for separating the hand from the background to exclude the effects of cluttered or dynamic backgrounds. 


\subsection{Hand Skeleton as Feature}

In this part we extract the features of the located hand. We use hand skeletons as features based on which we define and compare different hand poses. The skeleton is a graph that summarizes the shape of an object and can be employed as an efficient shape descriptor for object recognition and image analysis. In order to obtain the skeleton of the hand, the Voronoi Diagram algorithm [31] is applied on the boundary points of the segmented hand, followed by a pruning process to extract the main skeleton. Using this approach, defining new postures can be as easy as saving a snapshot of the posture into the system. In the HANDY framework, the user can perform the custom poses in front of the Kinect and the system saves the skeleton data of the specified poses for later use in hand pose and gesture recognition.

\subsection{Hand Pose Estimation}

Extracted features of the hand are analyzed in this part to estimate the hand pose. Hand pose estimation refers to the recognition of a single posture of the hand. Hand poses are defined based on their corresponding skeletons. In the proposed approach, each user is initially required to make a pose bank consisting of a series of reference hand poses which are used later for pose estimation based on a similarity measure. Dynamic Time Warping [32, an algorithm for comparing time series, is used for the similarity estimation of two skeletons. Skeleton points are ordered as DTW applies on mono-dimensional time series. Moreover, for a fair comparison, the skeletons are normalized to be transition and scale insensitive.

\subsection{Gesture Recognition}

The aim of this part of the system is to analyze the sequence of the hand pose features to recognize the performed gesture. The HANDY system is able to recognize generic (dynamic) gestures. Each gesture is represented by a sequence of hand poses defined in our hand pose bank. A gesture gets sampled during its performance and each sample frame is analysed and the hand pose is extracted and mapped to the closest hand pose in the pose bank using our pose estimation method. Due to the stochastic nature of gesture performance by the users, we adopted Hidden Markov Models (HMMs) to represent each gesture. It is worth noting that misrecognition of an intermediate hand pose and assignment of the closest hand pose from the bank is tolerated by the gesture recognition mechanism thanks to HMMs. Using HMMs for gesture recognition, training is needed before the system be able to recognize the gestures. In practice, the recognition system encodes a user gesture into a sequence of hand poses and calculates the probability that the sequence belongs to one of the trained gestures. The trained gesture which obtains the highest probability is chosen as the performed gesture. 


\section{Methodology}

We conducted two studies to evaluate our gesture recognition system regarding its effectiveness for older users. The rest of this section is dedicated to the explanation of these two studies.

\subsection{Experiment 1: Younger Participants}

Seven younger subjects without any hand problems participated in the first study (average age $=29, \mathrm{SD}=6.3$ ). The study took place in our lab environment. At the beginning a brief explanation of the system was given along with the necessary instructions on its use. The participants were guided to record 21 static hand poses in order to build up the hand pose bank and then train the system with a set of five more generic gestures. The participants were asked to perform 20 trainings for each gesture followed by 30 test gestures in order to evaluate the recognition accuracy. Gestures had a fixed length of 1 second, and were sampled 10 times during their performance. Figure 2 depicts a generic hand pose bank created by a participant. Five generic gestures are illustrated in Fig. 3 . The hand poses in the pose bank and the gestures were designed by the authors. Gestures contain key point poses in their performance which belong to the hand pose bank. In 30, we have explained in detail the technical aspects concerning the number and choice of hand poses in the pose bank as well as for the gestures. Finally, participants were asked to comment on the difficulty of hand pose shaping and gesture performance.

The recognition accuracy of the system was evaluated under two scenarios: 1) the system is trained and used by a single participant; 2) the system is trained by other participants and then used by another participant, so the training data of the whole set of participants is used for the gesture recognition.

\subsection{Experiment 2: Older Participants}

In the second study, 10 elderly people volunteered to participate ( 7 females, average age $=68.5, \mathrm{SD}=4.45)$. In the first part, they were asked to carry on a lighter version of the same test which had been done on younger subjects in order to evaluate how age affects the recognition accuracy of the system. Among

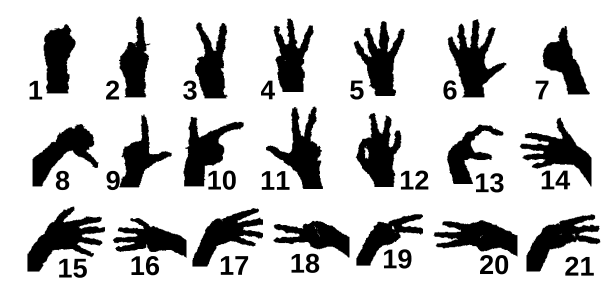

Fig. 2. Hand pose bank consisting of 21 static hand poses 


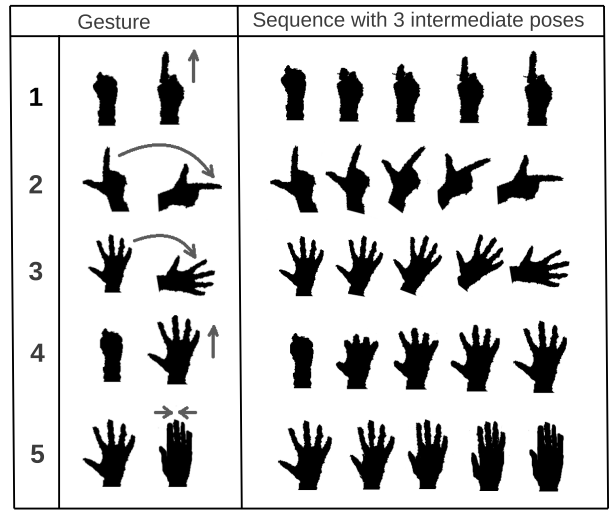

Fig. 3. Five gestures younger participants were asked to perform

the older participants we had one case of arthritis, one case of hand deformity, and one case of hand tremor. Like in the previous experiment, after explaining the purpose and potential applications of such gestural interaction system and providing the participants with required instructions on how to shape hand poses and how to perform gestures, they were asked to register 21 hand poses to create the pose bank, and then perform a set of 3 gestures instead of five with lower training sets. This was done to lessen the physical burden. In some cases, particularly hand deformity and arthritis, the final hand pose bank contained a reduced number of reference hand poses (minimum $=13$ ). The recognition accuracy of the system was evaluated just for the scenario where the system is used by single person. In case of hand tremor, the participant was asked to keep the pose for a certain amount of time ( 1 second in our tests) and more than 1 sample was taken from the hand pose (10 in our case). From these 10 samples, the most repeated hand pose was considered as the estimated hand pose. Using Hidden Markov Models was another alternative to tackle hand tremor: sampling the hand poses in 1 second and considering the hand pose as a gesture.

\section{Results and Discussion}

\subsection{Experiment 1: Younger Participants}

As mentioned before, a total of 7 younger adults participated and completed the test. No significant change was observed in the results. Table 1 summarises the obtained results for each participant and for each of the gestures (previously depicted in Fig. 3) in the first scenario in which the system is trained and used by a single user as well as for the second scenario where the system is trained by other participants and used by a new participants. Participants P6 and P7 were asked to use their left hands.

For the second scenario (S2) only the training sets of the 5 participants that used their right hand were considered. In average, slightly less accurate results 
were obtained in scenario $2(\mathrm{~S} 2: 96 \%)$ with respect to scenario 1 (S1:97\%). This suggests that with external training data for some universal gestures, initial training is not needed. Participants were asked to rank the gestures based on their difficulty. Gestures involving difficult hand manoeuvres like wrist rotation (gestures 2 and 3, see Fig. 3) were commented to be the most difficult ones.

Table 1. Recognition Accuracy (\%) for Experiment 1 - Younger Participants

\begin{tabular}{c|ll|ll|ll|ll|ll|}
\multicolumn{2}{c}{} & \multicolumn{2}{c}{ Gesture1 } & \multicolumn{2}{c}{ Gesture2 } & \multicolumn{2}{c}{ Gesture3 } & \multicolumn{2}{c|}{ Gesture4 } & \multicolumn{2}{c|}{ Gesture5 } \\
\cline { 2 - 11 } \multicolumn{1}{c|}{} & S1 & S2 & S1 & S2 & S1 & S2 & S1 & S2 & S1 & S2 \\
\hline P1 & 100 & 100 & 90 & 90 & 100 & 100 & 96.6 & 100 & 100 & 93.3 \\
P2 & 100 & 100 & 100 & 100 & 100 & 100 & 100 & 96.6 & 100 & 100 \\
P3 & 100 & 100 & 100 & 100 & 96.7 & 100 & 96.6 & 86.6 & 100 & 96.6 \\
P4 & 90 & 100 & 100 & 96.6 & 100 & 93.3 & 100 & 80 & 100 & 90 \\
P5 & 83.3 & 96.6 & 100 & 93.3 & 100 & 93.3 & 76.6 & 100 & 96.6 & 100 \\
P6 & 86.6 & - & 80 & - & 100 & - & 100 & - & 100 & - \\
P7 & 96.6 & - & 100 & - & 100 & - & 96.6 & - & 93.3 & - \\
\hline
\end{tabular}

\subsection{Experiment 2: Older Participants}

For the second study on older participants, 10 older persons volunteered to participate but only 6 of them completed the test (for reasons which will be mentioned). One of the participants was left-handed and thus performed the gestures with his/her left hand. Each test was done in one session. Most of the tests were done in an institute for elderly education (run by elderly themselves) and a few of them in our lab environment. Each test started with a general friendly talk describing the system and its potential applications setting a smooth start for the rest of the test. At the beginning, elderly participants were asked to specify their preferred interaction modality with technological artefacts and what applications they could think of using hand gestures. Five (out of 10) chose voice as their preferred interaction modality. They argued that this modality is easier to use and more natural. Three participants preferred traditional (keyboard, mouse) interaction modality. Having already learned how to use it, satisfaction of current small needs, and its tangibility and physical interaction were among the mentioned reasons. Finally, one participant chose touch interaction and one participant the gestural interaction. It is worth mentioning though that only this last participant was aware of potential applications of gestures having known TV sets controllable by hand gestures. When asked if they come up with any ideas for an application using hand gestures, most of them had a difficult time fantasizing one. A participant suggested it as a way to facilitate difficult manipulative tasks (like a door handle requiring much force to be turned). Another one mentioned mobility problems of the elderly and usage of gestures to issue commands from a fixed position.

In the next stage, we asked the participant to register the hand poses to create the pose bank. Required instructions were given orally; they needed to wave to 
draw the attention of the hand tracking mechanism and then keep the hand stable for a couple of seconds. Some participants (including the participant suffering from arthritis) mentioned that some hand poses were uncomfortable (specifically poses 4,20,21,15,17,19, see Fig. 2). The participants with hand deformity (suffering from Dupuytrens contractur) could perform only poses involving the first three fingers: if it would be possible to choose the input modality, he definitely prefers voice communication. He was not forced to go on with the test.

Afterwards, older participants were asked to complete a reduced version of the same gesture performance test done by younger participants. The number of gestures was reduced to 3 (gestures 1,2, and 5, see Fig. 3). Furthermore, rather than 50 training and test performances for each gesture, they were asked to do only 25 performances for training and test. The fatigue caused by the complete test had become evident in a mini pilot test. However, even with a lower number of required performances, three participants left the experiment at this point. We think this happened not only because of fatigue due to gesture performance but also because of lack of motivation. So, a more cheerful training phase through a game and/or having several test sessions could be beneficial. For the participant with hand tremor, the part of the system handling tremor was activated.

An average gesture recognition accuracy of $80 \%$ was achieved (compared to $90 \%$ range for younger participants). The main factor responsible for the lower recognition accuracy is less training numbers for elderly participants. Indeed, if we decrease the training number for younger participants, accuracy drops into the $80 \%$ range (similar to elderly results). Online training (training when the system is in use) could compensate for the lower numbers of training at the beginning. It is important to note that although hand problems like arthritis might lead to less accurate hand pose estimations but using HMMs diminishes these less accurate estimates at gesture performance level.

\section{Conclusions and Future Work}

In this work we proposed a vision-based hand gesture recognition system which is able to estimate user hand poses based on a personalized hand pose bank. Moreover our system is able to recognize generic hand gestures represented as stochastic sequences of hand poses modeled by HMMs. Furthermore, we conducted two experiments to investigate the potential benefits of our system for elderly people.

Our results show that our system is flexible enough to accommodate some of the physical limitations elderly people suffer from, mainly limitations related to an ageing hand, like hand tremor and arthritis. This was achievable in our system mainly thanks to personalizable hand poses and hand gestures. Thus, we think personalizability is an important ingredient of an effective gestural interaction system especially for users with specific needs.

There is still a lot to do to fill the gap between the technical requirements and human factors requirements for an effective, natural and inclusive gestural interaction system especially for specific users like the elderly. A gesture recognition 
system with high potentiality for personalization (flexible gesture definition with no or minimal decrease of accuracy) could be a suitable solution. The strength of personalization lies in the fact that it is a natural (and sometimes unconscious) way to adjust the gestural interaction to one's physical -and even cognitive- capabilities. This requires further investigation and is what we plan to examine in the future: evaluating the potential benefits of personalization on gestural interaction for specific target users like the elderly.

Acknowledgements. We would like to kindly thank Marina Peduzzi and Guido Tosatto for helping us organizing meetings and sessions with our elderly participants. We also deeply thank Auser Università Popolare Como for its collaboration with us.

\section{References}

1. UN: Department of economic and social affairs (desa) world population ageing 2009. DESA, United Nations, New York (2009)

2. UN: Review and appraisal of the progress made in achieving the goals and objectives of the programme of action of the international conference on population and development, 1999 report. United Nations publication, Sales No. E.99.XIII.16 (1999)

3. Kalache, A., Gatti, A.: Active ageing: a policy framework. Advances in gerontology $=$ Uspekhi gerontologii/Rossiiskaia akademiia nauk. Gerontologicheskoe Obshchestvo 11, 7-18 (2002)

4. Malanowski, N., Ozcivelek, R., Cabrera, M.: Active ageing and independent living services: the role of information and communication technology. European Communitiy (2008)

5. Rautaray, S.S., Agrawal, A.: Vision based hand gesture recognition for human computer interaction: a survey. Artificial Intelligence Review, 1-54 (2012)

6. Erol, A., Bebis, G., Nicolescu, M., Boyle, R.D., Twombly, X.: Vision-based hand pose estimation: A review. Computer Vision and Image Understanding 108(1), 52-73 (2007)

7. Karam, M.: PhD Thesis: A framework for research and design of gesture-based human-computer interactions. PhD thesis, University of Southampton (2006)

8. Wachs, J.P., Kölsch, M., Stern, H., Edan, Y.: Vision-based hand-gesture applications. Communications of the ACM 54(2), 60-71 (2011)

9. Fisk, A.D., Rogers, W.A., Charness, N., Czaja, S.J., Sharit, J.: Designing for older adults: Principles and creative human factors approaches. CRC press (2012)

10. Ranganathan, V.K., Siemionow, V., Sahgal, V., Yue, G.H.: Effects of aging on hand function. Journal of the American Geriatrics Society 49(11), 1478-1484 (2001)

11. Stößel, C., Wandke, H., Blessing, L.: Gestural interfaces for elderly users: help or hindrance? In: Kopp, S., Wachsmuth, I. (eds.) GW 2009. LNCS, vol. 5934, pp. 269-280. Springer, Heidelberg (2010)

12. Murthy, G., Jadon, R.: A review of vision based hand gestures recognition. International Journal of Information Technology and Knowledge Management 2(2), 405-410 (2009)

13. Camastra, F., De Felice, D.: LVQ-based hand gesture recognition using a data glove. In: Apolloni, B., Bassis, S., Esposito, A., Morabito, F.C. (eds.) Neural Nets and Surroundings. Smart Innovation, Systems and Technologies, vol. 19, pp. 159168. Springer, Heidelberg (2013) 
14. Garg, P., Aggarwal, N., Sofat, S.: Vision based hand gesture recognition. World Academy of Science, Engineering and Technology 49(1), 972-977 (2009)

15. Zhu, H.M., Pun, C.M.: Real-time hand gesture recognition from depth image sequences. In: 9th Int. Conf. Computer Graphics, Imaging and Visualization (2012)

16. Liu, X., Fujimura, K.: Hand gesture recognition using depth data. In: Proceedings of the Sixth IEEE International Conference on Automatic Face and Gesture Recognition, pp. 529-534. IEEE (2004)

17. Binh, N.D., Shuichi, E., Ejima, T.: Real-time hand tracking and gesture recognition system. In: Proc. GVIP, pp. 19-21 (2005)

18. Chen, F.S., Fu, C.M., Huang, C.L.: Hand gesture recognition using a real-time tracking method and hidden markov models. Image and Vision Computing 21(8), 745-758 (2003)

19. Starner, T., Weaver, J., Pentland, A.: Real-time american sign language recognition using desk and wearable computer based video. IEEE Trans. on Pattern Analysis and Machine Intelligence 20(12), 1371-1375 (1998)

20. Molina, J., et al.: Real-time user independent hand gesture recognition from timeof-flight camera video using static and dynamic models. Machine Vision and Applications 24(1), 187-204 (2013)

21. Baudel, T., Beaudouin-Lafon, M.: Charade: Remote control of objects using freehand gestures. Commun. ACM 36(7), 28-35 (1993)

22. Freeman, D., Vennelakanti, R., Madhvanath, S.: Freehand pose-based gestural interaction: Studies and implications for interface design. In: IHCI, pp. 1-6 (2012)

23. Lee, S.S., Chae, J., Kim, H., Lim, Y.K., Lee, K.P.: Towards more natural digital content manipulation via user freehand gestural interaction in a living room. In: Proc. UbiComp 2013, pp. 617-626 (2013)

24. Norman, D.A.: Natural user interfaces are not natural. Interactions $17(3), 6-10$ (2010)

25. Malizia, A., Bellucci, A.: The artificiality of natural user interfaces. Commun. ACM 55(3), 36-38 (2012)

26. Lee, T.-Y., Kim, H.-H., Park, K.-H.: Gesture-based interface using baby signs for the elderly and people with mobility impairment in a smart house environment. In: Lee, Y., Bien, Z.Z., Mokhtari, M., Kim, J.T., Park, M., Kim, J., Lee, H., Khalil, I. (eds.) ICOST 2010. LNCS, vol. 6159, pp. 234-237. Springer, Heidelberg (2010)

27. Sunwoo, J., Yuen, W., Lutteroth, C., Wünsche, B.: Mobile games for elderly healthcare. In: Proceedings of the 11th International Conference of the NZ Chapter of the ACM Special Interest Group on Human-Computer Interaction, pp. 73-76. ACM (2010)

28. Gerling, K., Livingston, I., Nacke, L., Mandryk, R.: Full-body motion-based game interaction for older adults. In: Proceedings of the 2012 ACM Annual Conference on Human Factors in Computing Systems, pp. 1873-1882. ACM (2012)

29. Anastasiou, D., Jian, C., Zhekova, D.: Speech and gesture interaction in an ambient assisted living lab. In: Proceedings of the 1st Workshop on Speech and Multimodal Interaction in Assistive Environments, pp. 18-27. Association for Computational Linguistics (2012)

30. Teimourikia, M., Saidinejad, H., Comai, S.: Handy: A configurable gesture recognition system. In: 7th Int. Conf. on ACHI (2014) (accepted for publication)

31. Aurenhammer, F.: Voronoi diagrams: A survey of a fundamental geometric data structure. ACM Computing Surveys (CSUR) 23(3), 345-405 (1991)

32. Berndt, D.J., Clifford, J.: Using dynamic time warping to find patterns in time series. In: KDD Workshop, Seattle, WA, vol. 10, pp. 359-370 (1994) 